\title{
El dilema sobre el coste-efectividad de la vacuna antineumocócica sigue abierto
}

\begin{abstract}
Ptreptococcus pneumoniae es una causa importante de morbi-mortalidad en nuestro medio. A pesar de la aparición de antibióticos de amplio espectro y de otros avances médicos, la mortalidad asociada a neumonía bacteriémica continúa siendo de un $25 \%$, sin haber experimentado cambios en los últimos 40 años. Además, Streptococcus pneumoniae, inicialmente sensible a la penicilina, se ha convertido en un germen con altas tasas de resistencia a la misma, constatándose también que entre el 30 y el $45 \%$ de los neumococos son resistentes a los antibióticos de uso más habitual.
\end{abstract}

El coste de tratar un número creciente de pacientes con enfermedad invasiva por cepas resistentes puede afectar de forma importante los recursos económicos de los servicios de salud, particularmente en aquellas comunidades donde exista un número importante de pacientes con alto riesgo de contraer enfermedad neumocócica ${ }^{1}$.

Datos declarados en España en el año 1996 señalan una incidencia de neumonía de 420/100.000 habitantes y año; siendo la tasa de mortalidad global por neumonías de 17 por 100.000 habitantes. Del total de neumonías, el $15-20 \%$ son causadas por neumococos con una letalidad del $5 \%{ }^{2}$. Además, el diagnóstico de infección neumocócica adquirida en la comunidad es difícil, ya que en los estudios realizados entre el 47 y el $58 \%$ de los casos no pudo determinarse la etiología. Si el hemocultivo o el cultivo de otro material normalmente estéril resulta negativo, la sensibilidad y la especificidad de otras pruebas diagnósticas, incluyendo las serológicas, no permiten un diagnóstico fiable ${ }^{1,3}$. Por ello, las dos maneras de conocer la incidencia de enfermedad neumocócica grave son, por un lado, el estudio de la enfermedad invasiva considerándose el estándar ideal de diagnóstico el aislamiento bacteriológico del neumococo en sangre $u$ otros medios habitualmente estériles como el líquido cefalorraquídeo, el líquido sinovial o el pleural. El otro método es determinar la incidencia de neumonía adquirida en la comunidad que ha requerido ingreso hospitalario y la estimación relativa de neumonía neumocócica.

En estudios realizados en Barcelona, Madrid y Valencia y que concuerdan con los resultados obtenidos en otros países europeos y los EE.UU., la incidencia observada fue de 57 casos por 100.000 habitantes $^{3,4}$. En nuestro país es un problema de salud pública y las ínfimas coberturas vacunales impiden conocer realmente la efectividad de la vacuna antineumocócica. La morbilidad y la mortalidad de la neumonía 
no bacteriémica es baja (3\%), pero se incrementa hasta el $25 \%$ en la enfermedad bacteriémica. Las estrategias de manejo de estos pacientes deben ir encaminadas a la prevención de enfermedad bacteriémica en aquéllos que presentan un mayor riesgo de padecer enfermedad neumocócica. ¿Cuáles serían los factores de riesgo asociados a infección neumocócica?: la edad, factores inmunológicos y otros factores precipitantes (Tabla I) ${ }^{1}$.

Tabla I

FACTORES DE RIESGO ASOCIADOS A ENFERMEDAD NEUMOCÓCICA

\section{FISIOLÓGICOS}

-Edad ( $<2$ años)

-Ancianidad

\section{DEFECTOS INMUNITARIOS}

-Deficiencias de anticuerpos primarias o secundarias: hipogammaglobulinemia, tumor de células $B, \ldots$

-Anormalidades de fagocitosis: hipo-a-esplenia, neutropenia, cirrosis hepática

-Déficits del complemento: ausencia de $\mathrm{C} 2$ o $\mathrm{C3}$

\section{FACTORES NO INMUNOLÓGICOS}

-Fractura de cráneo

-Disrupción del epitelio respiratorio (influenza, tabaco, otros tóxicos)

-Obstrucción de la trompa de Eustaquio

—Perfusión vascular disminuida: anemia falciforme, insuficiencia cardiaca congestiva

Se cree que la vacunación de las personas de 65 años y mayores con la vacuna antineumocócica es efectiva en la prevención de la enfermedad, pero la evidencia de la protección clínica permanece incierta ${ }^{5}$. A cualquier edad, la inmunodepresión es el mayor factor de riesgo para desarrollar la enfermedad. La incidencia de la enfermedad neumocócica invasiva en personas con el síndrome de inmunodeficiencia adqui- 
rida es de 800 a 1.400 casos por 100.000 personas. Ante el problema creciente del desarrollo de resistencias del neumococo a los antibióticos, la vacunación constituye una herramienta fundamental ya que es la única forma de prevenir la enfermedad ${ }^{6}$. Por otro lado, la utilización de la vacuna antineumocócica ha sido poco importante a nivel mundial y es únicamente en Estados Unidos donde, aún siendo bajo, el porcentaje de población diana que ha recibido la vacuna es mayor ${ }^{7} \mathrm{y}$ es por ello que son necesarios más estudios y un periodo más largo de seguimiento para resolver algunas de las dudas que se están planteando en torno a la utilidad real de la vacuna antineumocócica de polisacáridos.

Algunos trabajos, como el metaanálisis de Moore, Wiffen y Lipsky ponen de manifiesto la controversia que hay respecto a la eficacia de la vacuna y, sobre todo, respecto al coste-efectividad. En su publicación revisan la evidencia de la efectividad de las vacunas antineumocócicas polivalentes de polisacáridos en ensayos prospectivos randomizados y controlados comparando la efectividad de estas vacunas en sujetos inmunocompetentes con placebo y con aquéllos que presentan una alteración del sistema inmune. Para ello seleccionaron trece estudios de las características señaladas en los que además se reflejaran hechos clínicos relevantes como las tasas de neumonía global, neumonía neumocócica, de infección respiratoria baja, bacteriemia y muertes por neumonía. Concluyen que este tipo de vacunas son efectivas en la población inmunocompetente para disminuir la incidencia global de neumonías y el resto de las tasas mencionadas y que en cambio no existe evidencia de beneficio para la población con alguna alteración del sistema inmune ${ }^{8}$. Sin embargo, debido a las diferencias en la capacidad del sistema inmunitario para hacer frente a la infección neumocócica, la efectividad de estas vacunas varía según el tipo de inmunodepresión, por lo que estos ensayos deberían estar categorizados según las características de los pacientes estudiados para poder establecer comparaciones. La efectividad de la vacuna de polisacáridos debe de ser evaluada también separadamente en pacientes inmunocomprometidos y en ancianos, ya que el riesgo de la enfermedad y la respuesta inmunológica son diferentes en ambos casos ${ }^{6}$. Orqvist, en su comentario a dicho metaanálisis incide en que no se deben mezclar "circunstancias" con "poblaciones". Además, expresa su duda sobre el poder del metaanálisis para discriminar un verdadero efecto de la vacuna sobre la enfermedad invasiva neumocócica en ancianos cuando el riesgo relativo es de 0,53 con un amplio margen de intervalo de confianza $(0,14-1,94)$ y una incidencia de $50 / 100.000$ para dicha enfermedad.

Estas discrepancias no son nuevas; así en 1994 fue publicado otro metaanálisis de ensayos controlados randomizados donde se concluía que la vacuna antineumocócica de polisacáridos era eficaz en la preven- 
ción de neumonías neumocócicas bacteriémicas para los serogrupos incluidos en sujetos de bajo riesgo, pero no mostraba datos concluyentes para demostrar su eficacia en el grupo heterogéneo que se considera de alto riesgo ${ }^{9}$. En cambio, en otro metaanálisis ${ }^{10}$ sobre 13 ensayos clínicos randomizados o quasi-randomizados y que incluían 65.000 pacientes, se llegó a la conclusión de que la vacunación reducía el riesgo de infección sistémica en un $83 \%$ y que no existía evidencia de que fuese menos eficaz en ancianos, en pacientes institucionalizados o en aquellos que presentaban enfermedades crónicas asociadas.

La vacuna antineumocócica de polisacáridos lleva comercializada aproximadamente 20 años y el debate sobre su eficacia todavía persiste. Esto en parte es debido a que esta vacuna no fue objeto de ensayos controlados randomizados antes de su comercialización, por lo que sería fundamental que la nueva vacuna conjugada sea apropiadamente evaluada $^{8,12}$. Otros estudios ya sugirieron en su momento la necesidad de realizar estudios controlados que no se han realizado por dificultades logísticas, coste y problemas éticos ${ }^{9,12}$. En la revisión de Jain, Jain y Gal reclamaban la realización de un ensayo en pacientes inmunodeprimidos con una muestra suficientemente amplia para garantizar el poder estadístico y analizar el coste-beneficio de la vacunación en este grupo de pacientes ${ }^{9}$. En un ensayo randomizado controlado en Uganda no se encontró beneficio de dicha vacuna, pero en dos trabajos - un estudio de casos y controles y otro observacional de cohortes en los EE.UU.- se concluyó que la vacuna era eficaz, recomendándose en dicho país ${ }^{6}$.

En los ancianos, los estudios de efectividad de la vacuna antineumocócica de polisacáridos contra la neumonía no bacteriémica, no muestran beneficio, aunque las limitaciones para determinar si los casos de neumonía son producidos por neumococo, hacen difícil valorar el efecto de la vacuna sobre la neumonía neumocócica. Contra la enfermedad invasiva existen dos ensayos clínicos randomizados (Honkanen y Ortqvist) en los que la vacuna ofrece una eficacia del $55-80 \%$ en este grupo, aunque disminuye con la edad y el paso del tiempo.

En un estudio realizado en los EE.UU. asumiendo la eficacia de la vacuna sólo para la neumonía bacteriémica, se concluye que la vacuna es coste-efectiva9. En el reciente trabajo publicado sobre coste-efectividad en cinco países europeos, incluido España, indica que la vacunación sistemática de los ancianos es rentable en los países industrializados. En este trabajo, se asume una incidencia global de 50/100.000 y entre un 20 y un $40 \%$ de enfermedad invasiva con lo que las tasas de coste-efectividad eran menores de 12.000 ecus por cada año de vida ganado por calidad (QALY) en los 5 países. Para España se utilizaron los datos epidemiológicos extraídos del trabajo de Valencia y, asumiendo que la vacuna fuera igualmente efectiva para prevenir las neumonías invasivas y las no 
invasivas, costaría 10.511 ecus (1995) por QALY. Con estos datos, la administración de la vacuna sería coste-efectiva ${ }^{3,5}$. Sin embargo, si los datos de incidencia de la enfermedad no fueran los descritos en este trabajo, si no más cercanos a las cifras del Boletín Epidemiológico Nacional, se pondría en duda el coste-efectividad de la vacuna.

En pacientes institucionalizados, la literatura señala ${ }^{13,14}$ la eficacia de la vacuna para controlar junto con la antibioterapia los brotes de neumonia neumocócica y bacteriemia en pacientes no vacunados previamente.

En una revisión ${ }^{15}$ de 14 estudios (8 realizados en Europa y 4 en los EE.UU.) que evaluaban los costes económicos de la vacunación antineumocócica se pone de manifiesto, con las limitaciones que este tipo de estudios puede tener (no se trata de una enfermedad crónica, el tiempo de estudio es corto y la tasa de vacunación en personas de edad es baja), una relación coste-beneficio favorable en los casos de la aplicación de la vacuna en grupos de riesgo, sobre todo en personas mayores y en pacientes infectados por el VIH mientras que la vacunación en la población general no parece reportar este beneficio.

En nuestro país, donde las cifras de resistencia del neumococo a los antibióticos es elevada y la población anciana es creciente, es importante conocer, desde cada comunidad autónoma responsable de la política de vacunación, el impacto del problema antes de la toma de decisiones. La vacuna antineumocócica de polisacáridos es una vacuna segura, eficaz con sus limitaciones en ancianos y en algunos grupos de riesgo y sería razonable la vacunación de personas mayores de 65 años institucionalizadas y decidir sobre la vacunación masiva en este grupo de edad después de conocer la incidencia de la enfermedad con mayor exactitud, a pesar de la dificultad que esto representa. Recientemente, se comercializó en los EE.UU. una vacuna heptavalente conjugada para su utilización en niños. En la actualidad se evalúan vacunas basadas en antígenos para todos los neumococos como la proteína de superficie neumocócica A (PspA) o la adhesina de superficie neumocócica A (Psa A). Se debe favorecer el desarrollo de estas vacunas porque estudios epidemiológicos sugieren la variable epidemiología de la enfermedad neumocócica invasiva. Se supone que estas vacunas, al igual que las conjugadas, debido a su componente proteico inducen memoria inmunológica, que supondría una ventaja sobre las ya existentes. Estas vacunas deben ser evaluadas en adultos debido al potencial teórico en la prevención de las neumonías no bacteriémicas y a la limitada respuesta de las personas inmunocomprometidas a la vacuna de polisacáridos $^{6}$.

Mientras que no dispongamos de una vacuna al menos tan eficaz y segura como la vacuna antineumocócica de polisacáridos 23 -valente, deberemos seguir utilizándola en los casos en los que esté recomendada y 


\section{editorial}

según los recursos disponibles, en espera de nuevos estudios que nos aclaren definitivamente tanto la efectividad como el coste-efectividad de esta vacuna.

\section{J. Álvarez, M. A. Mayer}

Miembros del Grupo de Enfermedades Infecciosas del Programa de Actividades Preventivas y de Promoción de la Salud (PAPPS-semFYC)

Grupo de Enfermedades Infecciosas del Programa de Actividades Preventivas y de Promoción de la Salud (PAPPS-semFYC): M. J. Álvarez, M. A. Mayer, J. Gómez, A. Pareja, E. Comín, C. Bata lla, R. Piñeiro, V. Niño.

\section{BIBLIOGRAFÍA}

1. Obaro SK, Monteil MA, Henderson DC. The pneumococcal problem. BMJ 1996; 312: 1521-5.

2. Pareja Bezares A, Álvarez Pasquín MJ, Batalla Martínez C, Comín Bertrán E, Gómez Marco JJ, Niño Martín V, et al. Prevención de las enfermedades transmisibles. Aten Prim 1999; (supl 1): 99-117.

3. Fedson DS. Perspectivas para la vacunación antineumocócica de las personas de edad avanzada en España. En: Vacunas 2000. Barcelona: Ed. M. Campins \& F. Moraga. Prous Science, 2000.

4. Bayas JM, Vilella A, González A, Conesa A. Hospitalizaciones por neumonía en un hospital general. Med Clin (Barc) 1999; 159: 1773-868.

5. Ament A, Baltussen R, DuruG, Rigaud-Bully, de Graeve D, Ortoqt, et al Cost-effectiveness of pneumococcal vaccination of older people study in 5 western european countries. Clin Infect Dis 2000; 31 (2): 444-50.

6. Whitney CG. Vaccination against pneumococcal disease: current questions and future opportunities. Bio Med Central 2000; $1: 7$.

7. Fedson D. Pneumococcal vaccination in the United States and 20 other developed countries, 1981-1996. Clin Infect Dis 1998; 26: 1117-23.

8. Moore R, Wiffen PJ, Lipsky BA. Are the pneumococcal polysaccharide vaccines effective? Meta-analysis of the prospective trials. BMC Family Practice $2000 ; 1: 1$.

9. Fine MJ, Smith MA, Carson CA, et al. Efficacy of Pneumococcal Vaccination in Adults. A meta-analysis of randomized controlled trials. Arch Intern Med 1994; 154: 2666-77. 


\section{editorial}

10. Hutchison BG, Oxman A, Shannon H, Lloyd S, Altmayer CA, Thomas K. Clinical effectiveness of pneumococcal vaccine. Meta-analysis. Can Fam Physician 1999; 2381-93.

11. Jain A, Jain S, Gant V. Should patients positive for HIV infection receive pneumococcal vaccine? BMJ 1995; 310: 1060-2.

12. Hirschmann JV, Lisky BA. The pneumococcal vaccine after 15 years of use. Arch Intern Med 1994; 154: 373-7.

13. Nuorti JP, Butler JC, Crutcher JM, Guevara R, Welch D, Holder P, et al. An outbreak of multidrug-resistant pneumococcal pneumonia and bacteremia among unvaccinated nursing home residents. N Engl J Med 1998; 338: 1861-8.

14. Musher DM. Pneumococcal outbreaks in nursing homes. N Engl J Med 1998; 338: 1915-6.

15. Beutels PH. Evaluations economiques de la vaccination pneumococcique. Pressed Med 1998; 27: S33-8. 